\title{
Hournal of Caneer
}

2010; $1: 120-125$

Research Paper

(C) Ivyspring International Publisher. All rights reserved

\section{WT-I expression in a spectrum of melanocytic lesions: Implication for diiferential diagnosis}

\section{Luke S. Chung ${ }^{1 凶}$, Yan-gao Man², George P. Lupton ${ }^{1}$}

1. Department of Dermatopathology, Armed Forces Institute of Pathology and American Registry of Pathology, Washington DC 20306, USA

2. Department of Gynecologic and Breast Pathology, Armed Forces Institute of Pathology and American Registry of Pathology, Washington DC 20306, USA

$\triangle$ Corresponding author: Luke S. Chung, MD, Department of Dermatopathology, Armed Forces Institute of Pathology, 6825 $16^{\text {th }}$ Street, NW, Washington DC 20306, USA

Received: 2010.07.19; Accepted: 2010.08.20; Published: 2010.08.24

\begin{abstract}
A previous in vitro study revealed that Wilms's tumor I (WT-I) transcripts were detectable in 7 of 9 melanoma cell lines, but not in any of 5-normal melanocyte strains tested. Our current study assessed the expression levels of WT-I protein in clinical samples, to determine whether the expression levels of the WT-I protein may be used as a novel marker to assist differential diagnosis. Paraffin-embedded tissue sections from 15 cases of malignant melanomas and 25 cases of benign nevi were subjected to immunohistochemistry with a monoclonal antibody against the human WT-I protein. The expression levels of WT-I protein among normal, benign, and malignant melanocytes were semi-quantitatively assessed. Strong and uniform WT-I immunoreactivities were seen in all or nearly all tumor cells in both the junctional and dermal components of all malignant melanomas, and also in a vast majority of the tumor cells of Spitz's $(n=8)$, recurrent $(n=2)$, and junction $(n=2)$ nevi. Distinct WT-I immunoreactivities were also seen in some isolated individual tumor cells or tumor cell clusters in the junctional component of compound nevus (9) and in intradermal nevus (2). It is interesting to note that some isolated normal appearing melanocytes or cell clusters, and all morphologically distinct endothelial cells were strongly positive to WT-I. However, all tumor cells within the dermal component of compound $(n=9)$ or deep penetrating nevi $(n=1)$, or capsular nevus inclusion of lymph node (I) were devoid of WT-I expression. Our findings suggest that the expression level of the WT-I protein has no significant value in distinguishing between Spitz's nevi and malignant melanoma, but it may be a useful marker in differentiating between benign and malignant melanocytes within the dermal component. Our findings also suggest that aberrant WT-I expression may have oncogenic properties that promote the initiation and progression of melanocytic lesions.
\end{abstract}

Key words: Wilms' tumor 1 gene; Melanoma; Benign melanocytic lesions; Differential diagnosis

\section{Introduction}

The incidence and mortality of melanoma have steeply increased in the last century mainly due to the earlier occurrence of primary lesions in young people and late detection of biologically aggressive nodular melanomas [1-5]. Clinically, there are a variety of melanocytic lesions, including atypical Spitz's nevus, pigmented spindle cell nevus, deep penetrating nevus, and atypical combined nevus, that often morphologically and immunohistochemically mimic melanoma [6-11]. Therefore, there is an urgent need to 
identify novel biochemical and/or molecular markers that are melanoma-specific, to assist clinical differentiation, early detection, and intervention of melanoma.

A previous study revealed that Wilms' tumor 1 (WT-1) gene was differentially expressed in cultured human normal and malignant melanocytes [12]. With PCR analysis, WT-1 transcripts were detected in 7 of 9 melanoma cell lines but not in any of 5-normal melanocyte strains. With Northern blot analysis, steady-state WT- 1 mRNA levels were seen in 2 of 4 melanoma cell lines but not in any of the normal melanocytes [12]. Together, these findings suggest that the products of the WT-1 gene may be exclusively or preferentially expressed in malignant melanocytes.

Based on the above findings, our current study attempts to assess the expression status of WT-1 protein in normal, benign, and malignant melanocytes of clinical samples, to determine whether the expression levels of the WT-1 protein may be used as a novel marker to assist differential diagnosis among different melanocytic lesions in clinical samples.

\section{Materials and Methods}

Formalin-fixed, paraffin-embedded tissue blocks from 15 patients with malignant melanomas, and 25 with benign nevi (including 9 compound nevi, 8 Spitz's nevi, 4 junctional nevi, 2 intradermnal nevi, 1 deep penetrating nevus and 1 capsular nevus inclusion in a lymph node), were retrieved from the files of Armed Forces Institute of Pathology. Consecutive sections at $4-5 \mu \mathrm{m}$ thickness were cut, placed on positively charged slides, and subjected to morphological and immunohistochemical assessment. Immunohistochemical staining was conducted using our published protocol [13]. Briefly, sections were incubated at $60-70{ }^{\circ} \mathrm{C}$ for 1 hour, deparaffinized with xylene, and washed with ethanol and water. Deparaffinized sections were incubated in 1X antigen retrieval solution (Biocare Medical, Foster City, CA) overnight at 60-70 ${ }^{\circ} \mathrm{C}$, washed in water and phosphate-buffered saline (PBS), treated with 3\% hydrogen peroxide and normal serum, and incubated with a monoclonal anti-WT-1 antibody (6f-H2; Cat \#: CMC789; Cell Marque, Hot Springs, AR) in a dilution of 1:20 at room temperature for 3-4 hours. After incubation with the primary antibody, sections were washed with PBS, and sequentially incubated with the corresponding secondary antibody, avidin-biotin-peroxidase solution, and chromogen diaminobenzidine (Vector, Burlingame, CA). After the chromogen reaction, sections were counterstained with hematoxylin, washed in tap wa- ter, dehydrated in ascending concentrations of ethanol, cleared in xylene, and mounted for evaluation.

The expression status of WT- 1 among these lesions was independently examined by two investigators. A given cell was considered WT-1 positive if distinct chromogen coloration was consistently seen in its cytoplasm or nucleus in at least two duplicates of the same immunostaining procedure. The intensity of WT-1 immunostaining was graded as diffusible $(+)$, weak $(++)$, moderate $(+++)$, strong $(++++)$, and very strong $(+++++)$. The percentage of WT- 1 positive cells was grade as 0 (negative), $1+(<10 \%), 2+$ (about $30 \%)$, $3+($ about $50 \%), 4+($ about $75 \%$ ), and $5+$ (all or nearly all cells).

To assess the specificity of the immunostaining, different controls were used, which included: (1) the substitution of the primary antibody with the normal serum, (2) the omission of the secondary antibody and, (3) serial dilutions of the primary antibody. All the immunostainings was repeated at least twice under the same condition.

\section{Results}

All negative controls were completely devoid of distinct WT-1 immunoreactivity. In sharp contrast, all or nearly all malignant melanocytes in both the junctional and dermal component were strongly and uniformly positive for WT-1 (Fig 1a-1d). Similarly, a vast majority of the tumor cells of Spitz's, as well as recurrent and junction nevi (Fig 1e-1h) were positive for WT-1. It is interesting to note that some isolated normal melanocytes or cell clusters were strongly positive to WT-1 (Fig 2a-2d). These WT-1 positive normal-appearing melanocyte clusters often displayed some malignancy-associated changes, including the loss of polarity, focal disruption of the basement membrane, and morphological resemblance to malignant melanocytes (Fig 2a-2d). It is also interesting to note that all morphologically distinct endothelial cells were strongly positive to WT-1 (Fig 2e-2f). Distinct WT-1 immunoreactivities were also seen in some isolated individual tumor cells or tumor cell clusters in the junctional component of compound nevus and in intradermal nevus, whereas all tumor cells within the dermal component of compound or deep penetrating nevi, or capsular nevus inclusion of lymph node were devoid of WT-1 expression (Fig 2g-h). The sub-cellular localization of WT-1 expression is predominantly in the cytoplasm, which is consistent with that of our previous study in human breast tissues [13]. The rough percentage of WT-1 positive cells and intensity of WT-1 immunostaining are listed in Table 1. 

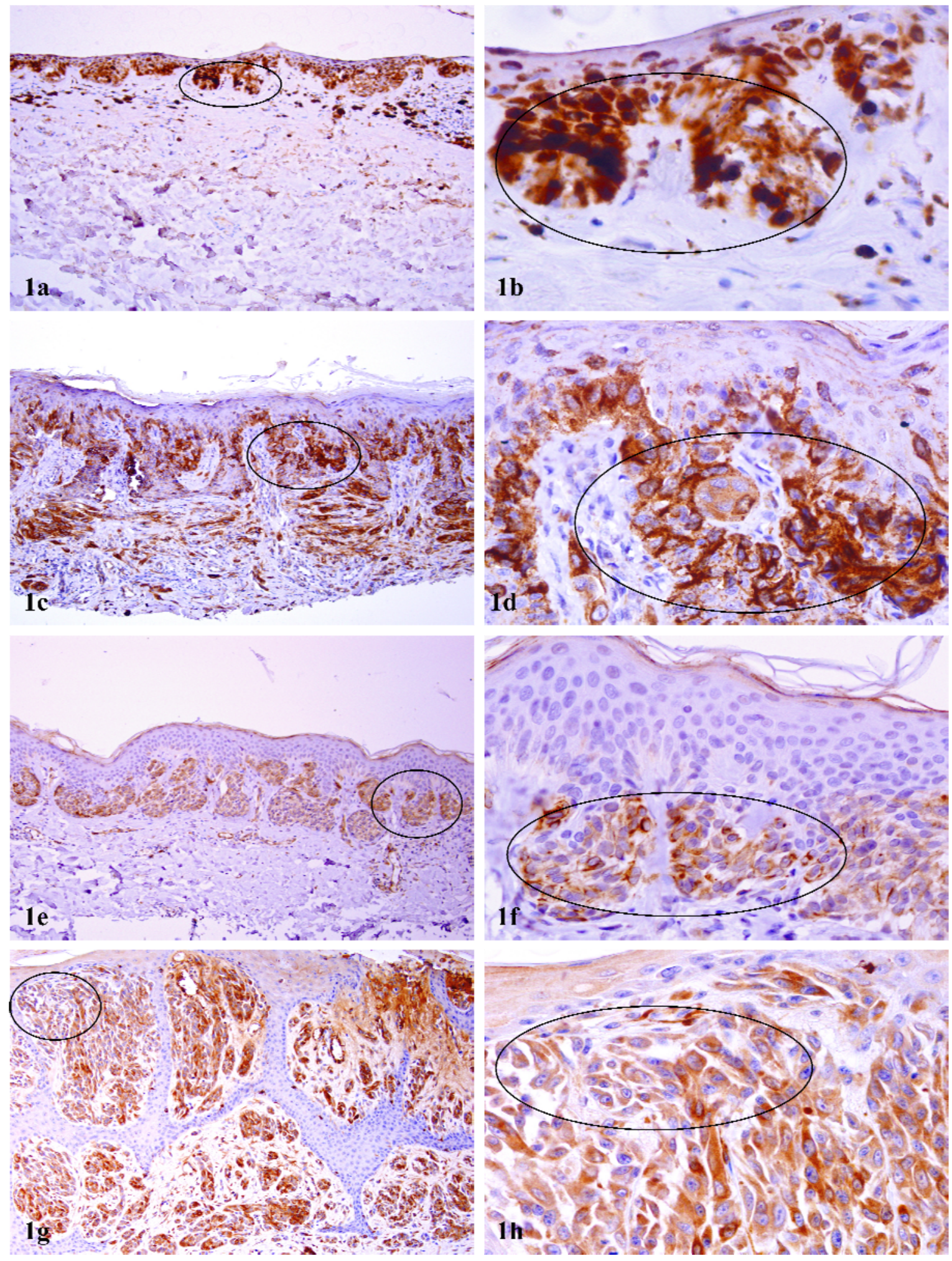

Fig I. WT-I expression in melanoma and Spitz's nevi. Paraffin-embedded tissue sections from patients with melanocytic lesions were immunostained for WT-I protein. Circles identify morphologically similar malignant and benign melanocytes with distinct WT-I immunoreactivities. Note that all or nearly all the tumor cells of in situ and spreading melanomas (a-d) were strongly and uniformly positive for WT-I. Similar WT-I immunostaining was also seen in all or nearly tumor cells in both the junctional and dermal components of Spitz's nevi (e-h). a, c, e, and g: I00X. b, d, f, and h: a higher magnification (300X) of a, c, e, and g, respectively. 

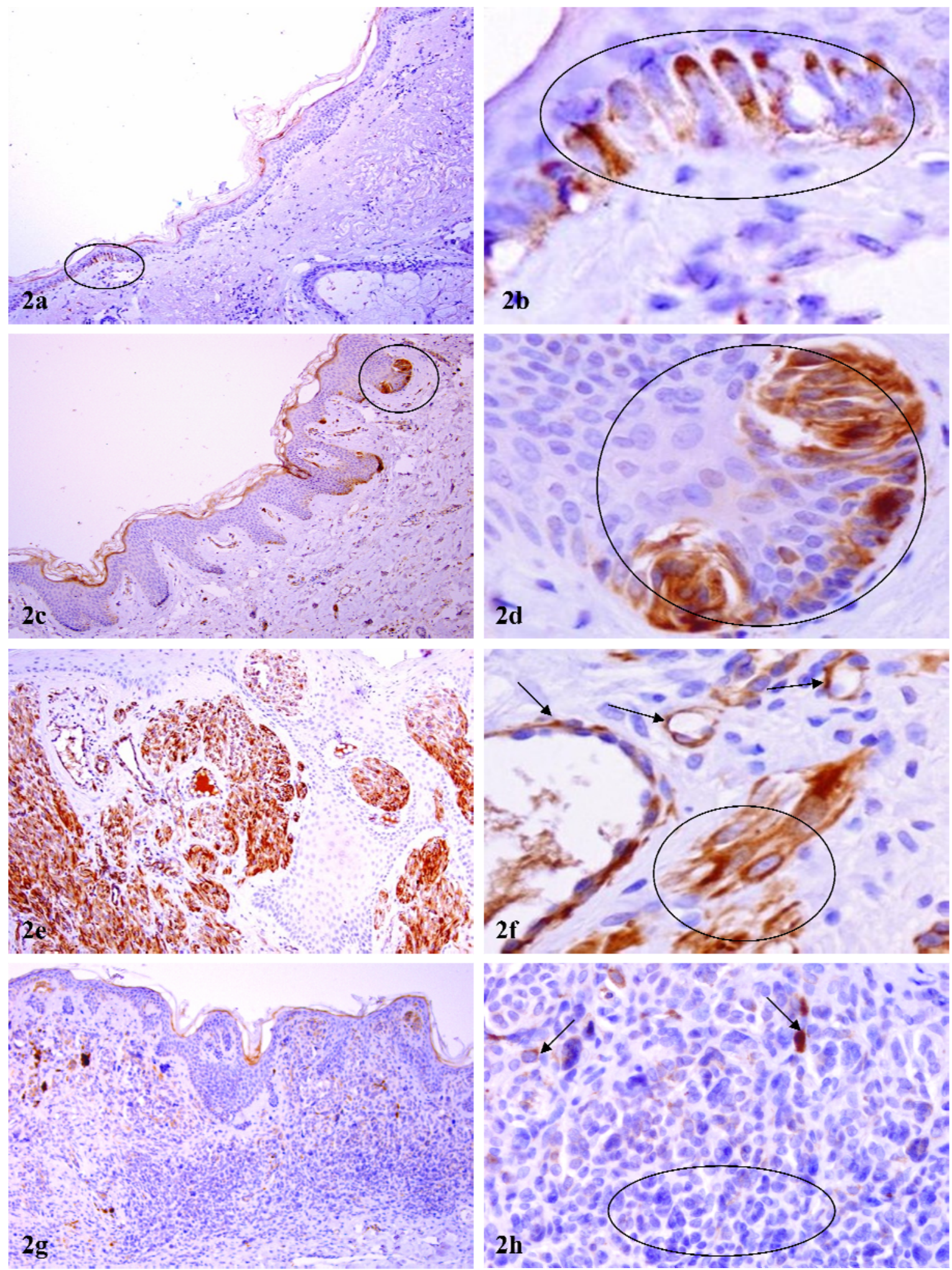

Fig 2: WT-I expression in normal appearing melanocytes and endothelial cells. Paraffin-embedded tissue sections from patients with melanocytic lesions were immunostained for WT-I protein. Circles identify melanocyte clusters with WT-I immunoreactivities in normal appearing tissues (a-d) and without WT-I immunoreactivities in the dermal component of compound or deep penetrating nevi. Arrows identify small blood vessels $(f)$ and isolated melanotyes $(h)$ with WT-I immunoreactivities. a, c, e, and g: I00X. b, d, f, and h: a higher magnification (300X) of a, c, e, and g, respectively. 
Table I. Comparison of WT-I Expression in different melanocytic lesions

\begin{tabular}{l|l|l|}
\hline Melanocytic lesion types & $\begin{array}{l}\text { Intensity and percentage of positive cells in } \\
\text { junctional component } \\
\text { Melanoma in situ (3) }\end{array}$ & $\begin{array}{l}\text { Intensity and percentage of positive cells in } \\
\text { dermal component }\end{array}$ \\
\hline Superficial spreading MM (2) & $+++++/++++$ & $\mathrm{NA}$ \\
\hline Nodular MM (8) & ++++++++++ & ++++++++++ \\
\hline Metastatic MM (1) & $\mathrm{NA}$ & +++++++++++ \\
\hline Desmoplastic MM (1) & $\mathrm{NA}$ & $++++/+++++$ \\
\hline Spitz's nevus (8) & $+++++/++++$ & +++++++ \\
\hline Recurrent nevus (2) & $+++++/++++$ & $\mathrm{NA}$ \\
\hline Junction nevus (2) & $+++++/++++$ & $\mathrm{NA}$ \\
\hline Compound nevus (9) & $+++++/+$ & $0 / 0$ \\
\hline Deep penetrating nevus (1) & $\mathrm{NA}$ & $0 / 0$ \\
\hline Intradermal nevus (2) & $\mathrm{NA}$ & $+/+$ \\
\hline Capsular nevus inclusion of & $\mathrm{N} / \mathrm{A}$ & $0 / 0$ \\
lymph node (1) & & \\
\hline
\end{tabular}

Intensity was graded as follows: 5+, strongest and 1+, weakest, and 0 , no staining. Sensitivity was graded as follows: 4+, diffuse staining of all melanocytes, $3+$, about $75 \%$ and $2+$, about $50 \%$ and $1+$, less than $25 \%$. NA-not applicable.

\section{Discussion}

The findings of our study appear to be in accordance with the findings of WT-1 staining in tissue cultures, in which melanoma cells are positive and normal melanocytes are negative for WT-1 [12]. However, the WT-1 expression is demonstrated not only in malignant melanoma in situ, superficial spreading melanomas, nodular melanomas, and metastatic melanomas, but also in Spitz's nevi, junctional nevi, particularly in recurrent nevi, and upper dermal melanocytes of benign nevi. These findings would suggest that differentiating between melanomas and atypical Spitz's nevus is not possible with WT-1 staining. Our findings are consistent with those of a recent study [14]. However, the WT-1 staining can be used as an adjuvant tool for differentiation between benign and malignant melanocytes within the dermal component. No definite conclusion, however, could be made at present, as the sample size of this study is too small.

It is interesting to note that a subset of isolated normal appearing melanocytes or melanocyte clusters are strongly positive for WT-1 (as those shown in Fig 2a-2d). Compared to their adjacent counterparts, these WT-1 positive normal-appearing melanocyte clusters generally display some malignancy-associated changes, including increased cell density, loss of polarity, focal disruptions of the basement membrane, and morphological resemblance to malignant melanocytes. It is also interesting to note that all or nearly all morphologically identifiable vascular structures show distinct WT-1 immunostaining (as those shown in Fig 2e-2f). The clinical significance of WT-1 expression in normal appearing melanosytes and endothelial cells is unknown. However, based on the fact that the expression level of WT-1 appear to increase with the increasing degree of malignancy, it is reasonable to speculate that aberrant WT-1 expression may have oncogenic properties that promote the initiation and progression of melanocytic lesions, and that the normal appearing melanosytes with strong WT-1 expression may represent the direct precursor of malignant melanoma. More importantly, as WT-1 is uniformly and strongly expressed in all malignant melanocytes and endothelial cells, the development of specific agents to manipulate the expression of WT-1 may have significant therapeutic value in malignant melanoma

\section{Notes}

The opinions and assertions contained herein represent the personal views of the authors and are not to be construed as official or as representing the views of the Department of the Army or the Department of Defense.

\section{Conflict of Interest}

The authors have declared that no conflict of interest exists.

\section{References}

1. Geller AC, Miller DR, Annas GD, Demierre MF, Gilchrest BA, Koh HK. Melanoma incidence and mortality among US whites. JAMA. 2002; 288: 1719-1720.

2. Rigel DS, Friedman RJ, Kopf AW. The incidence of malignant melanoma in the United States. J Am Acad Dermatol 1996; 34: 839.

3. Demierre M, Chung C, Miller D, Geller A. Early detection of thick melanomas in the United States. Arch Deramtol.2005; 141: 745-750.

4. Purdue MP, Freeman LB, Anderson WF, Tucker MA. Recent trends in incidence of cutaneous melanoma among U.S. Caucasian young adults. J Invest Dermatol. 2008; 128(12): 2905-2908.

5. Beddingfield FC3rd. The melanoma epidemic: res ipsa loquitur. Oncologist. 2003;8(5):459-65.

6. Shimek CM, Golitz LE. The golden anniversary of the Spitz's nevus. Arch Dermatol 1999; 135(3):333-335. 
7. Weedon D, Little JH. Spindle and epithelioid cell nevus in children and adults: a review of 211 cases of the Spitz's nevus. Cancer 1977; 40(1): 217-225.

8. Spatz A, Calonje E, Handfield-Jones S, et al. Spitz tumors in children: a grading system for risk stratification. Arch Dermatol 1999; 135(3): 282-285.

9. Barnhill RL, Flotte T, Fleischli M, Perez-Atayde A. Cutaneous melanoma and atypical Spitz tumors in childhood. Cancer 1995; 76: 1833-1845.

10. Barnhill RL, Mihm MCJr. Pigmented spindle cell nevus and its variant: distinction from melanoma. Br J Dermatol 1989; 121: 717-726.

11. Sau P, Graham JH, Helwig EB. Pigmented spindle cell nevus: a clinicopathologic analysis of minty five cases. J Am Acad Dermatol 1993; 28: 565-571.

12. Rodeck U, Bossler A, Kari C, et al. Expression of the WT1 tumor gene by normal and malignant human melanocytes. International Journal of Cancer. 1994; 59: 78-82.

13. Li JH, Man YG. Dual usages of single wilms' tumor 1 immunohistochemistry in evaluation of breast tumors. Cancer Biomarkers 2009; 5: 109-116.

14. Rosner K, Mehregan DR, Moussai D, Abrams J, Trump G, Mehregan DA. WT1 marker is not sufficient for distinguishing between melanoma and melanocytic nevi. J Cutan Pathol 2009; 36: 1077-1082. 